\title{
Contributing Reviewers in 2014
}

J Pediatr Intensive Care 2015;4:i-ii.

Journal of Pediatric Intensive Care wishes to recognize those who contributed as an expert peer reviewer of submitted scientific papers in 2014.

Thank you for your contributions to Journal of Pediatric Intensive Care in 2014.

Aslemo Abdo

Joanna Abraham

Ayobami Akenroye

Samin Alavi

Karel Allegaert

Howard Amital

Minoru Ando

Michael Beck

Robin A. Bernhoft

Laura A. Bonouvrie

Zara Brenner

Stefan Bushuven

Thiery Carrel

Desiree Caselli

Brian Casserly

Ming-Ren Chen

Kuo-Liang Chiang

Seth Corey

Abdallah R. Dalabih

Isıl Davarcı

Simon Davies

Korcan Demir

Khalil J. Diab

Brian C. Drolet

Christine N. Duncan

Yves Durandy

Christopher C. Dvorak

Jeffrey D. Edwards

Mohammed ElSheemy

Benedict Eneman

Kristin Engebretsen

İlkay Erdoğan

Rong Fan

Jun Feng

Francisco Fernandez-Carrion
Marino S. Festa

Emer Finan

Douglas Fraser

Andrew Fretzayas

Stuart H. Friess

Nikolaos Gatselis

Avihu Gazit

Nimeshan Geevasinga

Christopher R. Gilbertgonda

John Giuliano

David D. Gonda

Ricardo González

Marta González-Vicent

Kourosh Goudarzipour

Anne Greenough

Sandeep Grover

Imad Haddad

Grenville Peter Hadley

Volkan Hazar

Kwok Ho

Zane Horowitz

Steve Horton

Jacqui Jauncey-Cooke

Sonata Jodele

Pran Kar

Jyrson Klamt

Esther Knapp

Sergio Landa-Juárez

Daniel Levin

Hong Liu

Jo Lo

Greame Maclaren

Nathalie L. Maitre

Varuna Mallya

Seth Manoach
Clyde T. Matava

Jennifer McArthur

Judith Mercer

Timur Mese

Eleanor Mesler

Carlo Messina

Malgorzata Mizerska-Wasiak

Marta Moniz

Brian Moseley

Arlene Naranjo

Steve Neustein

Eric S. Nussbaum

Peter E. Oishi

Fatma Visal Okur

James Orlowski

Mirjam Osthoff

Ionnais Papassotiriou

Minal R. Patel

Luc Quintin

Sadeq Quraishi

Adrienne Randolph

Michael Reade

Corsino Rey

Darren Roberts

Ewa Romejko-Wolniewicz

Patrick Ross

Jonathan Roth

Antonio Ruggiero

Mohamed Saleem

Prakash Satwani

Jan Schieveld

Achim Schmitz

Kirk R. Schultz

Donna Seger

Dina Seif 
Henryk Siniawski

Angela Smith

Brandon Smith

Brent W. Snow

Duygu Sonmez-Duzkaya

Lauren Staggs

Vesna Stojanovic

Jeffrey W. Surcouf

Ru-Jeng Teng

Poonam P. Thankavel
Cody C. Trowbridge

Ozum Tuncyurek

Jennifer Tynan

Murat Ugurlucan

Amanda Ullman

Kiran K. Upadhyay

Celalettin Ustun

Subramanian Vaidyanathan

Josephus J.P. van Gestel

Marc van Heerde
Scott Welak

Wolfgang Wetsch

Jackson Wong

Shohei Yamamoto

Nadir Yehya

Deniz Yilmaz

Şebnem Yılmaz Bengoa

Warren Zuckerman

Hale Ören 